\title{
Rapid increase of carbapenemase-producing Klebsiella pneumoniae strains in a large Italian hospital: surveillance period 1 March - 30 September 2010
}

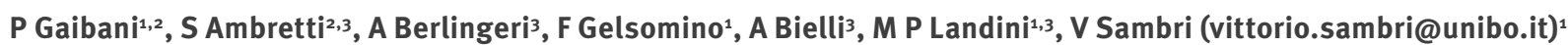

1. Regional Reference Centre for Microbiological Emergencies (CRREM), Bologna, Italy

2. These authors contributed equally to this paper

3. Bacteriology Section of the Operative Unit of Clinical Microbiology, S.Orsola-Malpighi University Hospital, Bologna, Italy

Citation style for this article:

Gaibani P, Ambretti S, Berlingeri A, Gelsomino F, Bielli A, Landini MP, Sambri V. Rapid increase of carbapenemase-producing Klebsiella pneumoniae strains in a large Italian hospital: surveillance period 1 March - 30 September 2010. Euro Surveill. 2011;16(8):pii=19800. Available online: http://www.eurosurveillance.org/ ViewArticle.aspx?Articleld $=19800$

Article published on 24 February 2011

The first case of carbapenemase-producing Enterobacteriaceae in Italy was reported in 2009. We performed a study over a period of seven months in 2010 to survey the circulation of Klebsiella pneumoniae carbapenemases (KPC) in a 1,500-bed university hospital in northern Italy and report the presence and rapid increase of these multidrug-resistant bacteria. The results raise a major concern about these pathogens and demonstrate the urgent need for infection control and antibiotic stewardship programmes.

\section{Introduction}

The spread of multidrug-resistant (MDR) gram-negative pathogens is one of the major hazards for patients requiring long-term hospitalisation or hospitalisation in intensive care units (ICU) [1]. In particular, given the use of carbapenems as second- or third-line drugs against MDR gram-negative germs, the resistance to this class of molecules poses a serious problem in the management of healthcare-associated infections. Acinetobacter baumannii and Pseudomonas aeruginosa, among the most common aetiologic agents of hospital-acquired infections worldwide, frequently show an MDR phenotype including resistance to carbapenem drugs. In the last few years, Klebsiella pneumoniae, often isolated from patients with pneumonia, bloodstream and urinary tract infections, has emerged worldwide as a carbapenemresistant microbe [2]. A resistance to carbapenems in Enterobacteriaceae can be mediated by three different mechanisms, namely: production of extended-spectrum beta-lactamase (ESBL) associated with loss of porins, production of metallo-beta-lactamase (MBL) and production of $K$. pneumoniae carbapenemases (KPC)type carbapenemases [3]. The first KPC-producing $K$. pneumoniae strain was isolated in 2001 in North Carolina [4] and until 2005 these MDR organisms were only identified along the eastern coast of the United States, where they rapidly became a frequent cause of hospital-acquired infections [4]. Since 2005, KPCproducing strains have been described worldwide [2].
As recently described [5], almost all European countries are affected by the expansion of carbapenem-resistant Enterobacteriaceae, even if the epidemiological scale of the diffusion is widely variable, from endemic presence, in particular in Greece, to sporadic occurrence. In Italy the first isolation of a KPC-positive K. pneumoniae was reported in $2009[6,7]$.

The aim of this study was to evaluate the incidence of $K$. pneumoniae strains showing a reduced susceptibility to carbapenems among patients hospitalised at the St.Orsola-Malpighi University Hospital in Bologna. The molecular mechanism of this phenotypic resistance was also investigated.

\section{Identification and characterisation of carbapenem-resistant Klebsiella pneumoniae strains}

The strain identification and antimicrobial susceptibility testing were performed using a Vitek2 automated system (Biomerieux, France). From 1 March to 30 September 2010, 431 consecutively isolated K. pneumoniae strains were included in this study. All the isolates showing a minimum inhibitory concentration (MIC) of $\geq 1 \mathrm{mg} / \mathrm{L}$ for meropenem (for this phenotype the Vitek2 system predicts probable production of KPC or MBL) were collected (86 isolates in total) and further evaluated in order to investigate the mechanism of resistance. During the seven-month surveillance period, at least one $K$. pneumoniae strain with suspected resistance to carbapenems was isolated from each of 69 patients for a total number of 86 strains. Additional antimicrobial testing was performed by E-test with Imipenem and Imipenem/EDTA (IPM-IPM/ EDTA) to detect the production of MBL, while the modified Hodge test was used as phenotypic confirmatory method for KPC-production [4]. This last method confirmed the production of carbapenemases in 52 strains isolated from 41 patients. Antimicrobial resistance associated with the production of MBL was excluded in all the collected isolates with a MIC of $\geq 1 \mathrm{mg} / \mathrm{L}$. The isolates were further analysed by PCR for the presence 
of genes controlling other resistance mechanisms [8]. The $b l a_{\mathrm{KPC}}$ gene was detected in 56 of the 57 isolates positive in the modified Hodge test. Among those, 45 resistance genes were $b a_{\mathrm{KPC}-3}$ gene and seven were $b l a_{\mathrm{KPC}-2}$ gene as determined by sequence analysis of the amplicons. Complete molecular genotyping is scheduled in order to better characterise and correlate all the KPC-positive strains.

\section{Clinical and epidemiological data}

All 52 KPC-positive strains were also resistant to all others beta-lactams (including the 3 rd and 4 th generation cephalosporines and piperacillin-tazobactam), to fluoroquinolones, and to sulfonamides. The susceptibility to gentamicin, tigecycline and colistin was retained in 47 of these KPC strains.

As shown in the Figure, most of the KPC-positive strains were isolated from urine (19 strains) and respiratory tract samples (nine isolates). Sixteen of the 40 patients bearing KPCs were hospitalised in an ICU (Figure, panel B). During the study period, the monthly number of new cases with KPCs and the rate of meropenem resistance increased from March to September,

\section{FIGURE}

Isolations of KPC-positive K. pneumoniae by anatomical site of isolation (A) and monthly cases by type of hospital ward (B), St.Orsola-Malpighi University Hospital, Bologna, 1 March - 30 September $2010(n=40)$
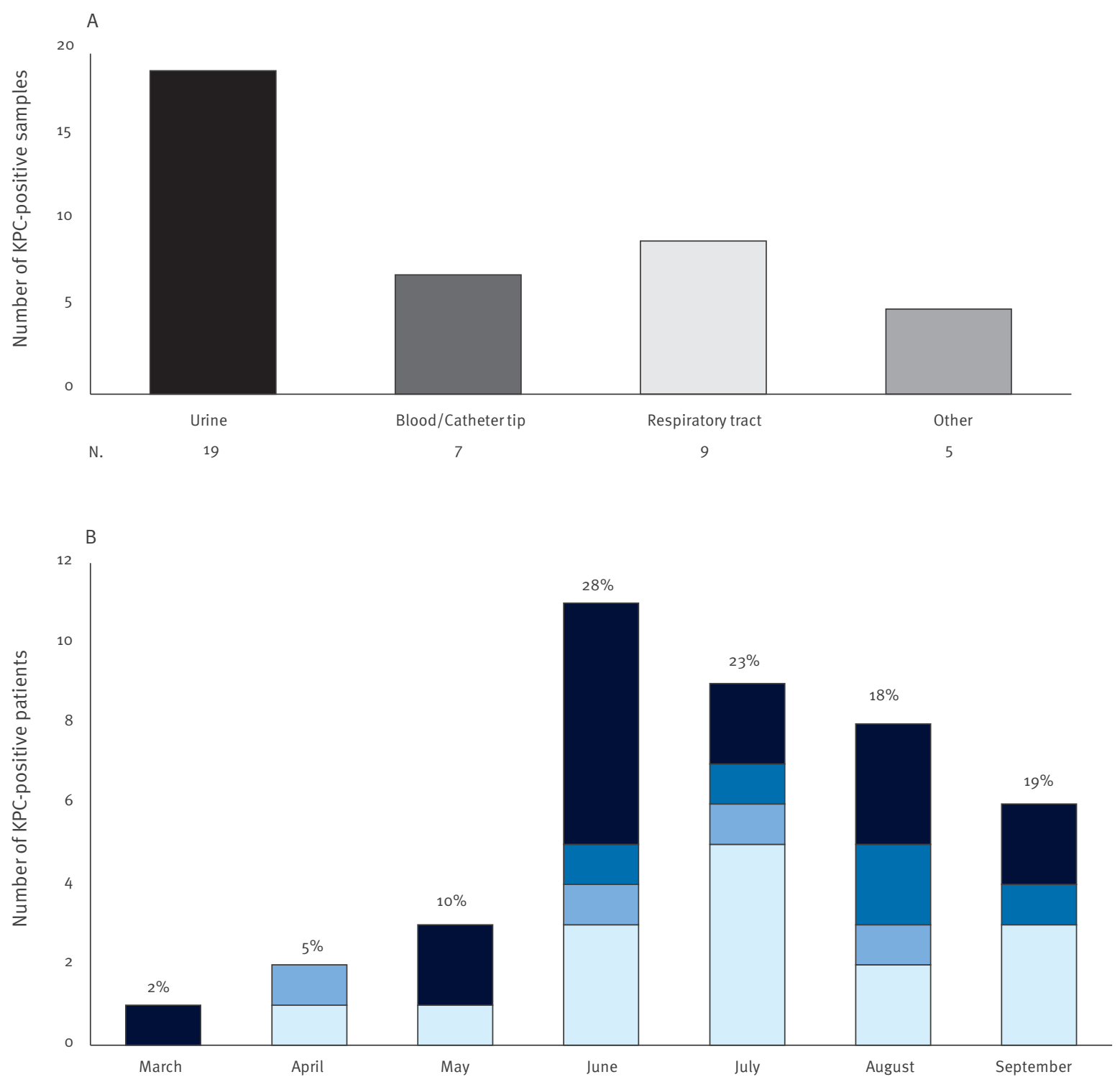

\begin{tabular}{lllllll}
\hline Intensive Care Unit & 1 & 0 & 2 & 6 & 2 & 3 \\
\hline Rehabilitation & 0 & 0 & 0 & 1 & 1 \\
\hline Surgical & 0 & 1 & 0 & 1 & 1 \\
$\square$ Medical & 0 & 1 & 1 & 3 & 2 \\
\hline
\end{tabular}

KPC: Klebsiella pneumoniae carbapenemase.

The monthly rates (\%) of meropenem resistance in $K$. pneumoniae isolates are shown above each bar (B). 
with a peak in June due to a cluster of colonisations and infections in an ICU (Figure, panel B).

\section{Discussion}

These data clearly demonstrate a consistent increase in carbapenem-resistant $K$. pneumoniae isolations during the study period. In addition our findings suggest that this phenomenon is linked to different KPC genotypes. It is noteworthy that in 2009 a similar surveillance protocol gave different results. In fact no KPC- or MBL- producing isolates were found at the time, suggesting that the phenotype with reduced susceptibility to carbapenems was mainly due to the production of ESBL associated with a loss in porins. In 2010 this phenomenon was largely replaced by KPC production that is nowadays the most prevalent cause of carbapenem-resistance in $K$. pneumoniae isolates. These data clearly indicate an increase of this phenomenon over a short period of time. It is interesting to note that KPCs in patients hospitalised in non-intensive or surgical wards were generally isolated only from urine whereas for ICU patients the main and first isolation site was the respiratory tract followed by other anatomical sites. We can speculate that this clinical feature is related to the different use of invasive devices during the hospitalisation: urinary catheters for medical and surgical patients, many other devices (intubation tubes, surgical drains, intravascular devices) for ICU patients.

From the microbiological point of view it is important to consider that more than $60 \%$ of KPC strains had MIC values of $2 \mathrm{mg} / \mathrm{L}$ for meropenem when evaluated by Vitek2: these isolates would be categorised as having intermediate susceptibility to meropenem using interpretation criteria from the Clinical and Laboratory Standards Institute (CLSI) after the revision of breakpoints in June 2010 [8]. If the breakpoints of the European Committee on Antimicrobial Susceptibility Testing (EUCAST) are applied [9], these isolates would be considered susceptible to meropenem. Our data suggest that a second level of investigation is required to evaluate the mechanism of reduced susceptibility, which could predict the clinical efficiency of carpapenem drugs. All the KPC-producing strains were still susceptible to antimicrobials that are not commonly used as alternative therapy for the treatment of nosocomial infections caused by to MDR gram-negative organisms [10]. In conclusion, the spread of carbapenem-non-suceptible Enterobacteriaceae in European countries a reason for great concern for public health services and calls for global diagnostic and management strategies. In our hospital in particular, KPCproducing $K$. pneumoniae strains spread fast and the isolation rate of these MDR bacteria is increasing. Appropriate surveillance and infection control measures are therefore urgently needed. We believe that it is also essential to apply strict antimicrobial stewardship policies to reduce the selective pressure that inevitably favours the emergence of carbapenem-resistant strains, so that these antibiotics remain therapeutically useful.
Acknowledgements

This study was supported by the Regione Emilia Romagna and by the University of Bologna.

References

1. Rice LB. The clinical consequences of antimicrobial resistance. Curr Opin Microbiol. 2009;12(5):476-81.

2. Nordmann P, Cuzon G, Naas T. The real threat of Klebsiella pneumoniae carbapenemase-producing bacteria. Lancet Infect Dis. 2009;9(4):228-36.

3. Queenan AM, Bush K. Carbapenemases: the versatile $\beta$-lactamases. Clin Microbiol Rev. 2007;20(3):440-58.

4. Lomaestro BM, Tobin EH, Shang W, Gootz T. The Spread of Klebsiella pneumoniae Carbapenemase-Producing K. pneumoniae to Upstate New York. Clin Infect Dis. 2006;43(3):e26-8.

5. Grundmann H, Livermore DM, Giske CG, Canton R, Rossolini GM, Campos J, et al. Carbapenem-non-susceptible Enterobacteriaceae in Europe: conclusions from a meeting of national experts. Euro Surveill 2010;15(46): pii=19711. Available from: http://www.eurosurveillance.org/ViewArticle. aspx?Articleld $=19711$

6. Giani T, D'Andrea MM, Pecile P, Borgianni L, Nicoletti P, Tonelli $F$, et al.. Emergence in Italy of Klebsiella pneumoniae sequence type 258 producing KPC-3 Carbapenemase. J Clin Microbiol. 2009;47(11):3793-4.

7. Ambretti S, Gaibani P, Caroli F, Miragliotta L, Sambri V. A carbapenem-resistant Klebsiella pneumoniae isolate harboring KPC-1 from Italy. New Microbiol. 2010;33(3):281-2.

8. linical and Laboratory Standards Institute (CLSI). Performance Standards for Antimicrobial Susceptibility Testing. Twentieth Informational Supplement M100-S20-June Update. Wayne, Pennsylvania: CLSI; 2010. Available from : http://www.clsi.org/ source/orders/free/m100-s20-u.pdf

9. European Committee on Antimicrobial Susceptibility Testing (EUCAST). Breakpoint tables for interpretation of MICs and zone diameters. Version 1.3. Växjö, Sweden: EUCAST; January 2011.. Available from: http://www.eucast.org/fileadmin/src/ media/PDFs/EUCAST_files/Disk_test_documents/EUCAST_ breakpoints v1.3 pdf.pdf

10. Livermore DM. Has the era of untreatable infections arrived? J Antimicrob Chemother. 2009;64(S1):i29-36. 